\title{
A NOTE ON THE COMPOSITIONS OF AN INTEGER
}

\author{
T.V. Narayana and G.E. Fulton
}

(received March 3, 1958)

1. Partial ordering of the $r$-compositions of $n$.

Given an integer $n$, we define an $r$-composition of $n$ as follows:

An $r$-composition of $n,\left(t_{1}, \ldots, t_{r}\right)$, is a set of $t_{i}$ where $t_{i} \geqslant 1$ is an integer for $i=1, \ldots, r$ such that

$$
t_{1}+\ldots+t_{r}=n .
$$

If $r$ is an integer such that $1 \leqslant r \leqslant n$, we have, obviously, $\left(\begin{array}{l}n-1 \\ r-1\end{array}\right)$ distinct $r$-compositions of $n$.

We shall say that an $r$-composition of $n,\left(t_{1}, \ldots, t_{r}\right)$, "dominates" the $r$-composition of $n,\left(t_{\}}, \ldots, t_{r}^{\prime}\right)$, if and only if

$$
t_{1} \geqslant t_{1}^{\prime}
$$$$
t_{1}+t_{2} \geqslant t_{1}^{\prime}+t_{2}^{\prime}
$$

$t_{1}+\ldots+t_{r-1} \geqslant t_{1}^{\prime}+\ldots+t_{r-1}^{\prime}$

Evidently

$$
t_{1}+\ldots+t_{r}=t_{1}^{\prime}+\ldots+t_{r}^{\prime}=n \text {. }
$$

The relation of domination defined by (A) is reflexive, transitive and anti-symmetric. It thus represents a partial ordering of the $r$-compositions of $n$.

We shall now make a transformation on the $r$-compositions of $n$, suggested by the relations (A). After this transformation,

Can. Math. Bull., vol. 1, no. 3, Sept. 1958 
we can decide immediately whether any two given $r$-compositions of $\mathrm{n}$ satisfy the relation of domination or not. Given an r-composition of $n,\left(t_{1}, \ldots, t_{r}\right)$, we associate with it the vector of r-elements $\left(\mathrm{T}_{1}, \ldots, \mathrm{T}_{\mathrm{r}}\right)$ obtained as follows:

$$
\begin{aligned}
\mathrm{T}_{1} & =\mathrm{t}_{1} \\
\mathrm{~T}_{2} & =\mathrm{t}_{1}+\mathrm{t}_{2} \\
& \cdot \\
& \cdot \\
\mathrm{T}_{\mathrm{r}-1} & =\mathrm{t}_{1}+\ldots+\mathrm{t}_{\mathrm{r}-1} \\
\mathrm{~T}_{\mathrm{r}} & =\mathrm{t}_{1}+\ldots+\mathrm{t}_{\mathrm{r}}=\mathrm{n} .
\end{aligned}
$$

We notice that the $T_{i}$ are integers and

$$
0<\mathrm{T}_{1}<\mathrm{T}_{2}<\ldots<\mathrm{T}_{\mathrm{r}}=\mathrm{n} \text {. }
$$

Evidently, given the composition $\left(t_{1}, \ldots, t_{r}\right)$, we can obtain the vector $\left(T_{1}, \ldots, T_{r}\right)$ and conversely, given the vector $\left(T_{1}, \ldots, T_{r}\right)$, (satisfying, of course, the relations $(B)$ ), we could obtain the $r$-composition $\left(t_{1}, \ldots, t_{r}\right)$. There are thus $\left(\begin{array}{c}n-1 \\ r-1\end{array}\right)$ "composition-vectors" $\left(T_{1}, \ldots, T_{r}\right)$ and we may, without fear of confusion, talk either of the $r$-composition $\left(t_{1}, \ldots, t_{1}\right)$ or the associated vector $\left(\mathrm{T}_{1}, \ldots, \mathrm{T}_{\mathrm{r}}\right)$.

If the composition $\left(t_{1}, \ldots, t_{r}\right)$ dominates $\left(t_{1}^{\prime}, \ldots, t_{r}^{\prime}\right)$ we shall find it convenient to say that the corresponding vector $\left(\mathrm{T}_{1}, \ldots, \mathrm{T}_{\mathrm{r}}\right)$ dominates the corresponding vector $\left(\mathrm{T}_{1}^{\prime}, \ldots, \mathrm{T}_{\mathrm{r}}^{\prime}\right)$. In the event that of two vectors, $\left(T_{1}, \ldots, T_{r}\right),\left(T_{1}^{\prime}, \ldots, T_{r}^{\prime}\right)$, neither is dominated by the other, we shall say that they are incomparable.

It can be proved by mathematical induction that the number of $\mathrm{r}$-compositions of $\mathrm{n}$ which are dominated by a particular $\mathrm{r}$ composition, whose vector is $\left(T_{1}, \ldots, T_{r}\right)$, is given by $D_{r-1}$ in the following formula:

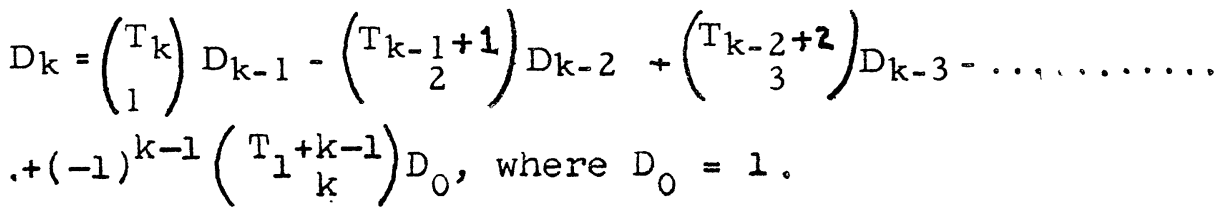


2. Lattice formed by the r-compositions of $\mathrm{n}$.

Given two vectors, $T=\left(T_{1}, \ldots, T_{r}\right), T^{\prime}=\left(T_{1}^{\prime}, \ldots, T_{r}^{\prime}\right)$ corresponding to the $r$-compositions of $n,\left(t_{1}, \ldots, t_{r}\right)$, $\left(t_{1}^{\prime}, \ldots, t_{r}^{\prime}\right)$ respectively, let

$$
\begin{aligned}
& M_{i}=\max \left(T_{i}, T_{i}^{\prime}\right) \\
& N_{i}=\min \left(T_{i}, T_{i}^{\prime}\right) \\
& \left(M_{r}=N_{r}=n\right)
\end{aligned}
$$

The vectors for all $\mathrm{i}=1, \ldots, \mathrm{r}$

$$
\begin{aligned}
& M=\left(M_{1}, \ldots, M_{r}\right) \\
& N=\left(N_{1}, \ldots, N_{r}\right)
\end{aligned}
$$

are easily seen to correspond to $r$-compositions of $n$, and we can prove easily that

(i) $\mathrm{M}$ dominates both $\mathrm{T}$ and $\mathrm{T}^{\prime}$.

(ii) If $\mathrm{V}$ dominates $\mathrm{T}$ and if $\mathrm{V}$ dominates $\mathrm{T}^{\prime}$, then $\mathrm{V}$ dominates $M$.

Thus $M$ is the l.u.b. of $T$ and $T^{\prime}$, and similarly $N$ is the g.1.b. of $\mathrm{T}$ and $\mathrm{T}^{\prime}$.

Let $T=\left(T_{1}, \ldots, T_{r}\right), T^{\prime}=\left(T_{1}^{\prime}, \ldots, T_{r}^{\prime}\right)$ and $T^{\prime \prime}=$ $\left(\mathrm{T}_{1}^{\prime \prime}, \ldots, \mathrm{T}_{\mathrm{r}}^{\prime \prime}\right)$ be the composition-vectors corresponding to any three $r$-compositions of $n$. Utilising the standard notation of lattice theory, we can easily prove that

$$
\left(T \cup T^{i}\right) \cap T^{\prime \prime}=\left(T \cap T^{\prime \prime}\right) \cup\left(T^{\prime} \cap T^{\prime \prime}\right) \text {; }
$$

for, this is equivalent to proving that, for all $i=1, \ldots, r$, $\min \left[\max \left(T_{i}, T_{i}^{\prime}\right), T_{i}^{\prime \prime}\right]=\max \left[\min \left(T_{i}, T_{i}^{\prime \prime}\right), \min \left(T_{i}^{\prime}, T_{i}^{\prime \prime}\right)\right]$ which is established by considering all possible relations between $\mathrm{T}_{\mathrm{i}}, \mathrm{T}_{\mathrm{i}}^{\prime}, \mathrm{T}_{\mathrm{i}}^{\prime \prime}$ such as:

$$
\begin{aligned}
& T_{i}<T_{i}^{\prime}<T_{i}^{\prime \prime} \\
& T_{i}=T_{i}^{\prime}<T_{i}^{\prime \prime} \\
& T_{i}<T_{i}^{\prime}=T_{i}^{\prime \prime} \text { etc. }
\end{aligned}
$$

We see that: 
THEOREM 1. The $r$-compositions of an integer $n$ form a distributive lattice. $(1 \leqslant r \leqslant n)$

3. An anti-isomorphism and an application.

Let $\mathrm{T}=\left(\mathrm{T}_{1}, \ldots, \mathrm{T}_{\mathrm{r}}\right)$ be the vector corresponding to an $r$-composition of $n$. Deleting the integers $T_{1}, \ldots, T_{r-1}$ from the set of positive integers $(1, \ldots, n)$ in their natural order, we have a set of $(n-r+1)$ integers which corresponds to the $(n-r+1)$-composition vector $T^{\prime}=\left(T_{1}^{\prime}, \ldots, T_{n-r+1}^{\prime}=n\right)$. It is clear that, if we start with the $(n-r+1)$-composition vector $T^{\prime}=\left(T_{1}^{\prime}, \ldots, T_{n-r+1}^{\prime}=n\right)$ and follow the above procedure, we arrive at the $r$-composition vector $T=\left(T_{1}, \ldots, T_{r}\right)$.

We have thus defined a one-to-one correspondence between the $r$-compositions and the $(n-r+1)$-compositions of $n$.

Let us consider the vectors $T^{(1)}=\left(T_{1}(1), \ldots, T_{r}(1)\right)$ and $T(2)=\left(T_{1}(2), \ldots, T_{r}(2)\right)$ as sociated with two distinct $r$-compositions of $n$, and the corresponding $(n-r+1)$-composition vectors $T^{(1)^{\prime}}=\left(T_{1}(1)^{\prime}, \ldots, T_{n-1+1}(1)\right.$ and $T^{(}(2)^{\prime}=\left(T_{1}(2)^{\prime}, \ldots, T_{n-r+1}^{(2)^{\prime}}\right.$. It is obvious that $T(2)$ dominates, is dominated by or is incomparable with $T(1)^{\prime}$ according as $T(1)$ dominates, is dominated by or is incomparable with $T(2)$, and hence:

THEOREM 2. The one-to-one correspondence between the $r$-compositions of $n$ and the $(n-r+1)$-compositions of $n$ is an anti-isomorphism.

Let $a(n)$ and $b(n)$ be the set of all compositions of $n$ with elements $\leqslant 2$ and $\geqslant 2$ respectively.

If an $\mathrm{r}$-composition of $\mathrm{n}$ involves the integers 1 and 2 only, the elements of the associated vector, $\left(T_{1}, \ldots, T_{r}\right)$, will be such that

and

$$
\mathrm{T}_{\mathrm{i}}-\mathrm{T}_{\mathrm{i}-1}=1 \text { or } 2 \text {, for } \mathrm{i}=2, \ldots, \mathrm{r} \text {, }
$$

$$
\mathrm{T}_{1} \quad-1 \text { or } 2 \text {. }
$$

Obviously, the elements of the corresponding $(n-r+1)$-composition vector, $\left(T_{1}^{\prime}, \ldots, T_{n-r+1}^{\prime}\right)$, will be such that

$$
T_{1}^{\prime}-T_{i-1}^{\prime} \geqslant 2 \text {, for } i=2, \ldots,(n-r) \text {, }
$$

while

$$
\begin{aligned}
\text { while } & \mathrm{T}_{\mathrm{n}-\mathrm{r}+1}^{\prime}-\mathrm{T}_{\mathrm{n}-\mathrm{r}}^{\prime} \geqslant 1 \\
\text { and } & \geqslant 1 \\
\mathrm{~T}_{1}^{\prime} & \geqslant 1 .
\end{aligned}
$$


To ensure that all elements of our $(n-r+1)$-composition are $\geqslant 2$, we add one to the first and last elements, giving us an $(n-r+1)$-composition belonging to $b(n+2)$. Clearly, starting with an $(n-r+1)$-composition of $b(n+2)$ and applying the above procedure in reverse, we obtain an $r$-composition of $a(n)$. Thus the anti-isomorphism of theorem 2 yields a one-to-one correspondence between the compositions belonging to $a(n)$ and the compositions belonging to $b(n+2)$.

A simple procedure for obtaining the composition of $b(n+2)$ corresponding to the composition of $a(n)$ is due to L.E. Bush. It can easily be seen that his procedure will give us the same one-to-one correspondence between the compositions belonging to $a(n)$ and the compositions belonging to $b(n+2)$.

\section{Acknowledgment}

This work was done while one of the authors was at the Summer Research Institute of the Canadian Mathematical Congress.

The equality of the number of compositions in $a(n)$ and $b(n+2)$ was posed as a problem in a recent Putnam examination. Professor Leo Moser suggested that there might be a one-to-one correspondence between these sets and we thank him and Professor Lambek for their interest in this note.

\section{References}

T.V. Narayana, C.R. Acad. Sci. Paris, (1955),1188-1189.

L.E. Bush, Amer. Math. Monthly, G4 (1957), 649-654.

McGill University

\section{EDITORIAL NOTE}

The correspondence given by L.E. Bush is in his report of solutions to problems on the Putnam examination. Such a correspondence was given earlier by $\mathrm{K}$. Bush. 